\title{
Preface to the 2nd Edition
}

The new edition of Mathematical Stereochemistry has been revised and rewritten by surveying recent advances during about 6 years after the publication of the first edition (2015). The most significant change is the addition of a new Chapter 13, which is entitled “Combined-Permutation Representations (CPRs) for GAP Calculation”. This chapter is concerned with the development of CPRs as computer-oriented representations of point groups, which enable us to treat reflection operations rationally in the GAP (Groups, Algorithms and Programming) system. This results in enhancing the applicability of Fujita's proligand method, Fujita's unit-subduced-cycle-index (USCI) approach, as well as Fujita's stereoisogram approach.

Soon after the publication of the first edition, I has discussed chirality (for supporting Le Bel's way to stereochemistry) and $R S$-stereogenicity (for supporting van't Hoff's way to stereochemistry) from a viewpoint of two kinds of handedness (S. Fujita, "Chirality and $R S$-Stereogenicity as Two Kinds of Handedness. Their Aufheben by Fujita's Stereoisogram Approach for Giving New Insights into Classification of Isomers”, Bull. Chem. Soc. Jpn., 89, 987-1017 (2016)). Experimental approaches (e.g., catalytic asymmetric synthesis by Ryoji Noyori (Nobel-Prize 2001)) have mainly relied on chirality, while approaches for nomenclature (e.g., the Cahn-Ingold-Prelog system by Vladimir Prelog (Nobel-Prize 1975) et al.) have mainly relied on stereogenicity proposed by Mislow et al. (not $R S$-stereogenicity). Fujita's $R S$-stereogenicity is differentiated from Mislow's stereogenicity by rational treatment of reflection operations to discuss the net interaction between chirality and stereogenicity, so that the $R S$-stereogenicity can be recognized as the second kind of handedness as compared with chirality as the first kind of handedness.

Soon after the publication of the first edition, I have also reported an account article entitled "Half-Century Journey from Synthetic Organic Chemistry to Mathematical Stereochemistry through Chemoinformatics” (S. Fujita, Iranian J. Math. Chem., 7, 155-221 (2016)). This article has stated three phases of my career, which have been summarized by respective monographs:

- Synthetic organic chemistry for investigating sterically-hindered molecules (that is to say, aziridines, heterophanes, and so on) and for developing sterically-enhanced dye-releasers of instant color photography (S. Fujita, "Organic Chemistry of Photography”, Springer-Verlag, Berlin-Heidelberg (2004) xix+587 pages).

- Chemoinformatics for retrieval of organic reactions by means of imaginary transition structures (ITSs) as newly-developed representations (S. Fujita, “ComputerOriented Representation of Organic Reactions”, Yoshioka-Shoten, Kyoto (2001) $\mathrm{x}+371$ pages).

- Mathematical stereochemistry for Fujita's USCI approach (S. Fujita, "Symmetry and Combinatorial Enumeration in Chemistry”, Springer-Verlag, Berlin-Heidelberg (1991) x+368 pages; S. Fujita, “Diagrammatical Approach to Molecular Symmetry 
and Enumeration of Stereoisomers”, University of Kragujevac, Faculty of Science, Kragujevac (2007) x+206 pages), for Fujita's proligand method (S. Fujita, “Combinatorial Enumeration of Graphs, Three-Dimensional Structures, and Chemical Compounds”, University of Kragujevac, Faculty of Science, Kragujevac (2013) xiv + 576 pages), and for Fujita's stereoisogram approach as a compresensive investigation on mathematical aspects of stereochemistry (S. Fujita, "Mathematical Stereochemistry” (the 1st Edition), De Gruyter, Berlin (2015) xviii+437 pages).

As a result, the three phases may relate to "STEM (Science, Technology, Engineering, and Mathematics) Education", which has been promoted as an educational reform project on a national scale during a decade in Europe and the USA, and now in Japan.

- S[cience] As an embodiment of S[cience], the design of dye releasers for instant color photography has been based on the fact that a sterically bulky tert-butyl group brings about the enhancement of dye-releasing efficiency.

- $\quad \mathbf{T}[$ echnology] and E[ngineering] With respect to the synthetic processes of these dye releasers, the reaction retrieval system based on the ITS concept supports the selection of effective synthentic routes. The processes of choosing candidate compounds (T[echnology]) and practical syntheses of them (E[ngineering]) can be regarded as an embodiment of T[echnology] and E[ngineering], so as to be claimed in several patents (e.g., S. Fujita, K. Koyama, Y. Inagaki, and K. Waki, US Patent 4336322 (1982)).

- M[athematics] Selection of suitable substituents for candidate compounds is concerned with combinatorial enumeration, where Fujita's proligand method for gross enumerations and Fujita's unit-subduced-cycle-index (USCI) approach for symmetry-itemized enumeration have been developed. These enumeration procedures require group-theoretical studies, esp. on point groups and permutation groups. For further discussions on stereochemisty, point groups and permutation groups have been integrated into stereoisomeric groups via $R S$-stereoisomeric group by means of Fujita's stereoisogram approach. This phase can be regarded as an embodiment of M[athematics].

After referring to STEM, it should be emphasized again that the newly-added Chapter 13 aims at further enhancement of mathematical capabilities of "Mathematical Stereochemisty", where the GAP (Groups, Algorithms, Programming) system, freely available from internet, is adopted as a versatile computer system for supporting group theory. The GAP system lays stress on enumeration of graphs on the basis of permutation groups. In order to enumerate 3D structures on the basis of point groups, the functions of considering ligand reflections should be added to the conventional procedures of the GAP system. For this purpose, combined-permutation representations (CPRs) 
of point groups have been developed. Thereby, Fujita's proligand method for gross enumerations and Fujita's unit-subduced-cycle-index (USCI) approach for symmetryitemized enumeration can be conducted with considering ligand reflections by means of GAP system. Moreover, this course of solution results in the integration of point groups and permutation groups under the GAP system, so as to produce versatile procedures for dealing with Fujita's stereoisogram approach.

All drawings in this book were prepared using the X'MTEX system, which was developed by myself and now available from the Comprehensive $\mathrm{T}_{\mathrm{E}} \mathrm{X}$ Archive Network (CTAN) https : //www . ctan.org/pkg/xymtex. The on-line manual of the X'MTEX system Version 5.01 (S. Fujita, "XYMTEX: Reliable Tool for Drawing Chemical Structural Formulas”, Shonan Institute of Chemoinformatics and Mathematical Chemistry, Kanagawa (2013), xvi+760 pages) is available from the CTAN site and also from my home page: http://xymtex.com/fujitas3/xymtex/indexe.html. In addition to detailed descriptions on how to use the X'MT: $\mathrm{MT}_{\mathrm{E}} \mathrm{X}$ system, the whole format of this manual provides us with an illustrative example for publishing a book containing both chemical structural formulas (S[cience]) and mathematical equations (M[athematics]). Because this new edition of Mathematical Stereochemistry has been typeset by $\mathrm{X}^{\mathrm{T}} \mathrm{MT}_{\mathrm{E}} \mathrm{X}$ incorporated in $\mathrm{T}_{\mathrm{E}} \mathrm{X} / \mathrm{AT}_{\mathrm{E}} \mathrm{X}$, it is also an illustrative example for publishing a book containing both chemical structural formulas (S[cience]) and mathematical equations (M[athematics]). 